\title{
EMI Risk Estimation for System-Level Functions Using Probabilistic Graphical Models
}

\author{
Lokesh Devaraj \\ HORIBA MIRA Limited \\ Nuneaton, UK \\ lokesh.devaraj@horiba-mira.com
}

\author{
Alastair R. Ruddle \\ HORIBA MIRA Limited \\ Nuneaton, UK \\ alastair.ruddle@horiba-mira.com
}

\author{
Alistair P. Duffy \\ De Montfort University \\ Leicester, UK \\ apd@dmu.ac.uk
}

\begin{abstract}
In general, the functions provided by complex systems often involve multiple sub-systems and components that are functionally dependent on each other. The dependency could be to receive power, control signals, input data, memory storage, feedback etc. With the increasing use of electronic systems to perform critical functions, the potential for malfunctions due to electromagnetic interference need to be identified and mitigated. Hence, a risk analysis, estimating the likelihood and severity of electromagnetic interference effects, is desirable from the very early stages of system development. In this paper, the use of probabilistic graphical models for estimating the likelihood of electromagnetic disturbances causing system malfunctions with various degrees of severity is demonstrated using a very simple case study. Statistical data are synthesised to illustrate the construction of conditional probability distribution tables for a Bayesian Network system model. Factorization and inference techniques are then applied to demonstrate the formulation and answer of queries that could be of value during system risk assessment.
\end{abstract}

Keywords - risk analysis, probabilistic graphical models, Bayesian network, failure analysis, electromagnetic interference.

\section{INTRODUCTION}

In the current practice of electromagnetic compatibility (EMC) in relation to road vehicles, the immunity testing conditions (i.e. frequency range and steps, field strength, exposure time, illumination direction, operational state etc.) are prescribed by regulatory standards. Some manufactures verify the immunity of their systems at more stringent levels for higher confidence. However, due to technical and economic limitations, it is impossible to test all possible electromagnetic interference conditions, resulting in an insufficiency of confidence due to epistemic uncertainties (i.e. things that could be known in principle, but in practice are not).

To achieve EMC for modern complex systems with rapid advancement in the features and functionality (like automated driving in road vehicles), the current industrial practice of rule-based EMC testing approach needs to be shifted, more towards a risk-based approach. As also discussed in [1], in order to accomplish a risk-based electromagnetic (EM) approach, hazards identification and risk estimation activities should commence from the initial concept phase of the system itself, and should be continuously refined as more and more system knowledge is obtained.

In [2], some of the risk factors to be considered to determine the risk parameters (likelihood and severity) for a

The research leading to these results has received funding from the European Union's Horizon 2020 research and innovation programme under the Marie Skłodowska-Curie grant agreement No 812790 (MSCA-ETN PETER). This publication reflects only the authors' view, exempting the European Union from any liability. Project website: http://etn-peter.eu/. system-level EM risk analysis are given. This includes estimation of the likelihood of EM interference (EMI) having an impact with a particular severity level for any system function, reflecting the functional dependence or interaction between the various components that are involved. The level of risk then depends on the severity of the consequences and the likelihood of their occurrence [3]. In this paper, the application of a probabilistic graphical model (PGM) for EMI risk analysis is illustrated by considering EMI to be a common-cause of failure for multiple components performing a system function. This analysis is carried out using a Bayesian network (BN) [4]. The significant increase in the application of BN models for risk analysis and dependability for complex systems is evident from [5]. However, there is very little literature on the application of PGMs for EM risk analysis considering EM interference to be a common-cause failures and cascading failures [6].

This paper provides a simple demonstration of systemlevel EMI risk estimation with PGMs, using a case study consisting of functionally dependent components as discussed in Section II. Further, the details of the EMI analysis procedure and results obtained for the impact analysis and risk estimation are given in Section III-IV. Integration of the simulation results to construct the conditional probability distribution (CPD) tables of the Bayesian network model and relevant inferences obtained therefrom are discussed in Section V. The merits and limitations of PGMs for EM risk analysis are summarised in Section VI. Finally, the conclusions and related future work are summarized in the last section. In addition, the Appendix provides some further details of derivation of the EMI probability distributions.

\section{SimPle CASE STUDY}

A complex system usually employs more than one component or subsystem (comprising sensors, controllers and actuators) to perform a system function. EMI that is present in the system environment can cause malfunctions by affecting more than one component, resulting in a common-cause failure. Additionally, due to functional dependence, any erroneous signal provided by one component/subsystem could be further passed to its dependent components/subsystems, causing much severe consequences at a system level [7].

For the purposes of this study, it is imagined that a hazard analysis has been carried out and a critical system function has been identified that depends on particular data signals that are at risk from possible corruption by EMI. Although errors of up to $20 \%$ in value of the parameter carried by these signals are tolerable, differences of $20-60 \%$ would be a nuisance and anything more than $60 \%$ is considered to be unacceptable. In order to assess the associated risk, it would be desirable to estimate the probability of EMI-induced errors reaching the identified severity levels. 
The critical data is assumed to be processed by a simple sub-system comprised of components $\mathrm{C} 1$ and $\mathrm{C} 2$ (for data transmission), and subsystems $\mathrm{S} 1$ and $\mathrm{S} 2$ (data processing) as shown in Fig. 1, is taken as an example to apply the BN model for analysing the likelihood of common-cause and cascaded failures due to EMI. The system model is based on the assumptions below.

- The system function is to deliver a pulse width modulated (PWM) representation of a sinusoid of frequency $F$ to subsystem $\mathrm{S} 2$, where $F$ is initially supplied as a digital signal to $\mathrm{C} 1$.

- Any deviation to the frequency of the sinusoidal wave reconstructed from the PWM wave by $\mathrm{S} 2$ is considered a malfunction.

- Subsystem S1 receives a numerical value transmitted via cable $\mathrm{C} 1$ in the form of a digital signal $m_{1}$.

- A sinusoidal signal with frequency equal to the received numerical value is then converted to a PWM wave $m_{2}$ by $\mathrm{S} 1$ and transmitted through $\mathrm{C} 2$ to subsystem $\mathrm{S} 2$.

- For the purposes of this case study, S1 and S2 are assumed to be immune to the EMI applied for the analysis, whereas $\mathrm{C} 1$ and $\mathrm{C} 2$ are both considered to be potentially susceptible to the EMI.

Component $\mathrm{C} 2$ is therefore functionally dependent on $\mathrm{C} 1$, as the frequency of the PWM signal transmitted through $\mathrm{C} 2$ is based on the value received by $\mathrm{S} 1$ from $\mathrm{C} 1$.

Assuming PWM modulation and demodulation functions denoted by $\operatorname{Pwm}\left(m_{j}\right)$ and $\operatorname{Ipwm}\left(m_{j}\right)$, respectively, are applied to a signal $m_{j}$, then the modulated signal $m_{2}=\operatorname{Pwm}\left(m_{1}\right)$ corresponds to a sinusoid of frequency $F$ if $\mathrm{C} 1$ is unaffected by EMI. Furthermore, if $\mathrm{C} 2$ is also unaffected by EMI then the demodulated signal $\operatorname{Ipwm}\left(m_{2}\right)$ has a frequency equal to $F$. However, if either or both signals are affected by EMI then a malfunction, where $\operatorname{Ipwm}\left(m_{2}\right) \neq F$, is a possible outcome.

\section{LIKELIHOOD ESTIMATION FOR EMI}

To emulate the susceptibility of $\mathrm{C} 1$ and $\mathrm{C} 2$ to EMI noise, continuous sinusoids of the form $N_{k}=A_{k} \sin \left(2 \pi f_{k} t+\varphi_{k}\right)$ were added to the cable signals $m_{1}$ and $m_{2}$. A total of 6262 random noise samples were derived, with uniform distributions over the ranges allocated for the amplitude $A_{k}(1$ to $1.5 \mathrm{~V})$, the frequency $f_{k}(15 \mathrm{kHz}$ to $0.3 \mathrm{MHz})$, and the initial phase $\varphi_{k}(0$ to $2 \pi$ radians $)$ of the noise.

\section{A. Generating Signals for $C 1$ and $C 2$}

The signals $m_{1}$ and $m_{2}$ that are transmitted through cables $\mathrm{C} 1$ and $\mathrm{C} 2$, respectively, are generated as outlined below.

1. The intended target value, $F$, is converted to a digital signal in binary format $(0=-1 \mathrm{~V}$ and $1=+1 \mathrm{~V})$, with a bit rate of $100 \mathrm{kbits} / \mathrm{sec}$. For a target value $F=150$,

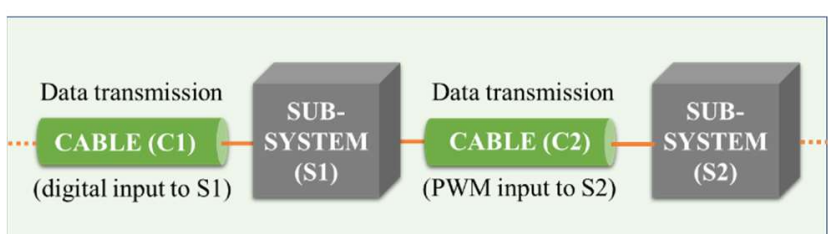

Fig. 1 System model considered for casestudy: sub-system S1 transmits input data (digital) through cable $\mathrm{C}$ 1, which is processed by sub-system $\mathrm{S} 2$ to provide the output data (PWM wave) through cable C2. the digital input to cable $\mathrm{C} 1$ corresponds to 10010110.

2. Where required, EMI may be added to the signal $m_{1}$.

3. Using an intersective method (a sawtooth waveform was used as the modulating waveform), a PWM pulse train is constructed as the input to $\mathrm{C} 2$, corresponding to a sinusoidal signal with frequency equal to the value (in $\mathrm{kHz}$ ) received by subsystem $\mathrm{S} 1$ in the form of $m_{1}$.

4. Where required, EMI may be added to the signal $m_{2}$.

5. The PWM pulse train that is transmitted via $\mathrm{C} 2$ is demodulated by $\mathrm{S} 2$ to retrieve the frequency of the sinusoid represented by $m_{2}$.

Signals were generated for three EMI conditions, with EMI applied individually to $m_{1}$ (denoted $\left.E_{1}\right)$ and $m_{2}\left(E_{2}\right)$, as well as applied to both $m_{1}$ and $m_{2}$ concurrently $\left(E_{3}\right)$.

\section{B. Probability of Malfunction}

If the frequency decoded by S2 under EMI condition $E_{n}$ is $G_{k}\left(E_{n}\right)$, then the corresponding relative deviation $D_{k}\left(E_{n}\right)$ from the target value $F$ is obtained from:

$$
D_{k}\left(E_{n}\right)=100\left|\frac{G_{k}\left(E_{n}\right)-F}{F}\right|(\%)
$$

The decoded frequency values $G_{k}\left(E_{n}\right)$ were obtained by selecting the highest peak $(\mathrm{Pk})$ of the Fourier transform $(\mathrm{Ft})$ of the signal demodulated by S2 for the three potential EMI conditions:

$$
\begin{gathered}
G_{k}\left(E_{1}\right)=\operatorname{Pk}\left\{\operatorname{Ft}\left[\operatorname{Ipwm}\left\{\operatorname{Pwm}\left(m_{1}+N_{k}\right)\right\}\right]\right\} \\
G_{k}\left(E_{2}\right)=\operatorname{Pk}\left\{\operatorname{Ft}\left[\operatorname{Ipwm}\left\{\operatorname{Pwm}\left(m_{1}\right)+N_{k}\right\}\right]\right\} \\
G_{k}\left(E_{3}\right)=\operatorname{Pk}\left\{\operatorname{Ft}\left[\operatorname{Ipwm}\left\{\operatorname{Pwm}\left(m_{1}+N_{k}\right)+N_{k}\right\}\right]\right\}
\end{gathered}
$$

The results of relative deviations due to EMI in C1 (2), C2 (3), and $\mathrm{C} 1$ and $\mathrm{C} 2$ concurrently (4) were obtained by adding the 6262 EM noise samples for each of the three cases. The data collected was used to generate the cumulative distribution functions (CDFs) for EMI that are illustrated in Fig. 2, for EMI in $\mathrm{C} 1$, in $\mathrm{C} 2$, and in both $\mathrm{C} 1$ and $\mathrm{C} 2$ concurrently (denoted C1\&C2). Details of the corruption of the signals $m_{1}$ and $m_{2}$ due to EMI are not key to the focus of this paper and are therefore explained in more detail in the Appendix, with relevant examples.

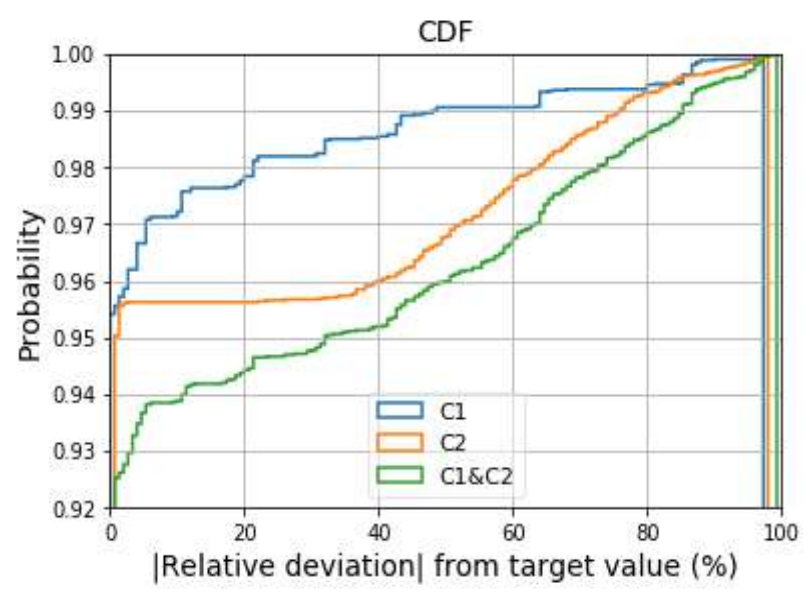

Fig. 2. Cumulative distribution functions for the deviations $D_{k}\left(E_{n}\right)$ due to EMI noise in $\mathrm{C} 1, \mathrm{C} 2$ and $\mathrm{C} 1 \& \mathrm{C} 2$. 


\section{SEVERITY OF EMI IMPACT AND RISK}

To enable estimation of the system level risk associated with the EMI, the relative deviation caused by the EMI is assumed to have varying levels of severity of consequences. Hence, based on the magnitude of the relative deviation, D (\%) from the intended target value, the severity $\mathrm{S}$ of the possible malfunctions is categorized as, low (tolerable), medium (undesirable) or high (intolerable) according to:

$$
S(D)=\left\{\begin{array}{cl}
\text { low } & \text { if } 0 \leq D \leq 20 \\
\text { medium } & \text { if } 20<D \leq 60 \\
\text { high } & \text { if } D>60
\end{array}\right.
$$

Probability values for the severity levels (low, medium or high) associated with EMI effects on $\mathrm{C} 1, \mathrm{C} 2$, and $\mathrm{C} 1 \& \mathrm{C} 2$ are given in Table I. A comparison of these probabilities shows that it is highly likely that the EMI considered here will be of low severity for the system function by affecting components $\mathrm{C} 1$ and $\mathrm{C} 2$ independently, with the result that the associated risk levels are also low, given that the estimated probabilities for low severity impacts are very high: $\mathrm{P}(\mathrm{C} 1=$ low $)=0.9784$ and $\mathrm{P}(\mathrm{C} 2=$ low $)=0.9562($ see Table I $)$.

From a component supplier's perspective, having such low risks might seem to be acceptable at first sight. However, it should be noted that the probability for having the low severity outcome for the $\mathrm{C} 1 \& \mathrm{C} 2$ situation $\mathrm{P}(\mathrm{C} 1 \& \mathrm{C} 2=$ low $)$ is smaller. This implies that, at a system-level, there is an increase in the likelihood of system malfunctions with medium and high severity, due to the functional dependence of components $\mathrm{C} 1$ and $\mathrm{C} 2$ (although in this particular example the increase is relatively small).

\section{EMI RISK ESTIMATION WITH BN}

For complex systems exploiting a high proportion of electrical and electronic components to perform system functions, EMI could be a common-cause of malfunctions or failures. The estimation of risk for such systems (i.e. determining the likelihood and severity of the EMI impacts) becomes increasingly difficult as the number of system functions when using more traditional tools (such as failure mode and effects analysis, fault trees, event trees etc.), , system components, and component failure modes increases. In this section, the application of EM risk analysis with BN (a PGM) is illustrated for the simple case study considered in this paper.

A BN is a graphical structure consisting of a network of nodes representing model variables and edges representing a causal relationship between pairs of connected nodes. The functional dependence between components can be considered as a causal factor in determining the likelihood of impacts at a particular level of severity for the system function. Considering the simple case study reported here, it is possible to represent the functional dependence of $\mathrm{C} 2$ on $\mathrm{C} 1$ in delivering the system function by using the BN graph and its associated conditional probability tables (CPDs), which are illustrated in Fig. 3.

TABLE I. Probability Distribution TABLE FOR SEVERITY OF EMI IMPACT FOR C1, C2, AND C1\&C2

\begin{tabular}{lccc}
\hline \hline & low & medium & high \\
\hline $\mathrm{C} 1$ & 0.978441 & 0.012137 & 0.009422 \\
$\mathrm{C} 2$ & 0.956244 & 0.021559 & 0.022197 \\
$\mathrm{C} 1 \& \mathrm{C} 2$ & 0.944267 & 0.023315 & 0.032418 \\
\hline \hline
\end{tabular}

P(C1) \begin{tabular}{|c|c|c|}
\hline C1 $1=$ low & C1 $=$ medium & $\mathrm{C} 1=$ high \\
\hline 0.978285 & 0.012135 & 0.00958 \\
\hline
\end{tabular}

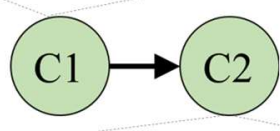

\begin{tabular}{|l|c|c|c|}
\hline \multicolumn{1}{|c|}{$\mathbf{P}(\mathbf{C} 2 \mid \mathbf{C} 1)$} & $\mathrm{C} 2=$ low & $\mathrm{C} 2=$ medium & $\mathrm{C} 2=$ high \\
\hline $\mathrm{C} 1=$ low & 0.963767 & 0.01322 & 0.023013 \\
\hline $\mathrm{C} 1=$ medium & 0.105263 & 0.855263 & 0.039474 \\
\hline $\mathrm{C} 1=$ high & 0.016667 & 0.2 & 0.783333 \\
\hline
\end{tabular}

Fig. 3. Simple BN representation of the functional dependence between components $\mathrm{C} 1$ and $\mathrm{C} 2$ as a causal factor to determine the probability of the degree of EMI impact on system function. Conditional distribution tables for EMI impact on $\mathrm{C} 1$ and $\mathrm{C} 2 \mid \mathrm{C} 1$ are given for nodes $\mathrm{C} 1$ and $\mathrm{C} 2$.

\section{A. CPD Table Entries for BN Nodes}

The estimated probability values in the CPD tables for BN shown in Fig. 3 were obtained by analysing the statistical data outlined in section III. As component $\mathrm{C} 1$ does not have any parent nodes, the probability distribution of $\mathrm{P}(\mathrm{C} 1)$ is independent of any other node variables (i.e. $\mathrm{C} 1$ is not dependent on any other component within the system to perform the system function). Hence, $\mathrm{P}(\mathrm{C} 1)$ is not conditional on any other node variable and is directly obtained from Table I. However, C2 is dependent on its parent node $\mathrm{C} 1$ and is therefore conditioned on the state space assigned to the node variable $\mathrm{C} 1$.

In Fig. 4, an event tree is used to illustrate the steps followed in determining the values of CPD table P (C2 $\mid \mathrm{C} 1)$ for node $\mathrm{C} 2$ in Fig. 3. It should be noted that, among the 6262 simulated noise samples initially considered for determining the CPD of EMI impact $\mathrm{P}(\mathrm{C} 2 \mid \mathrm{C} 1)$, only the specific event $\mathrm{P}(\mathrm{C} 2=$ low $\mid \mathrm{C} 1=$ high $)$ was never observed. Assigning zero probability for a rare but possible event only because it was not observed in a samples study would be unacceptable due to its impact on related probability values. In order to overcome this situation, a Bayesian approach to estimate the probability using equivalent sample size is given in [9]. For the case study, no prior knowledge is available to determine the required sample size to observe this event and its probability can only be quantified as $<1 / 6262$. To avoid the ambiguity of assigning a zero-probability value to a possible event, it is assumed that it would be observed if noise sample 6263 were to be generated (an indication of the additional sample is given by the red circles in Fig. 4).

The CPD values of node $\mathrm{C} 2$ in the $\mathrm{BN}$ provides the likelihood of system level impact due to EM susceptibility of component $\mathrm{C} 2$, given each state space of the node variable $\mathrm{C} 1$. For instance, if the EM noise affecting component $\mathrm{C} 1$ caused a medium level impact, then the probability that the impact is high due to EM susceptibility of C2 is approximately 4\% (see Fig. 3).

\section{B. Joint Probability of EMI Impact Severity}

As previously given in [2], if all the nodes of a $\mathrm{BN}$ are responsible for a system-level function, then the overall probability for the functional deviation at system-level can be determined from their joint probability distribution (JPD). In this case study, both nodes $(\mathrm{C} 1$ and $\mathrm{C} 2)$ in Fig. 3 are assigned 


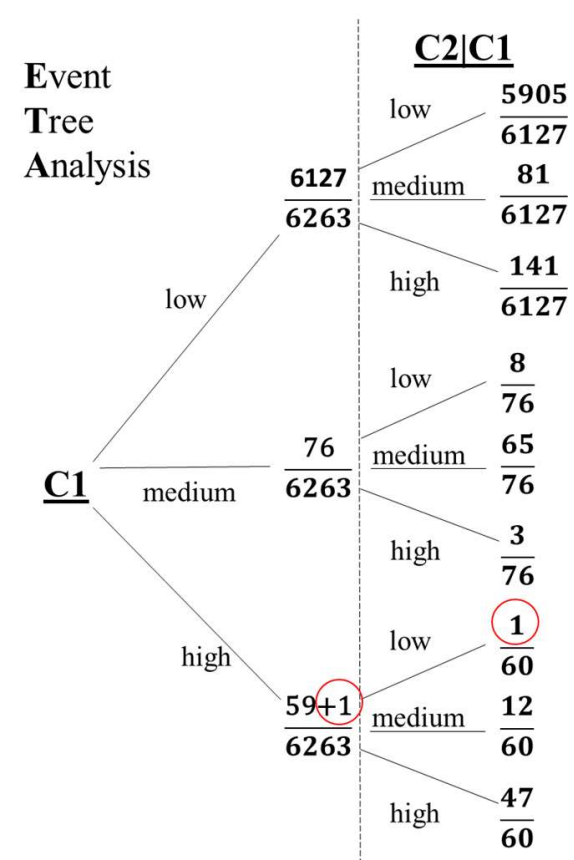

Fig. 4. An event tree to illustrate the condition probability values obatained for the CPD table in Fig. 3.

to perform the system-level function, hence the overall probability measure of system-level impact can be determined from their JPD, which is given by:

$$
P(C 1, C 2)=P(C 1 \mid p a\{C 2\}) P(C 2 \mid p a\{C 2\})
$$

where the function $\mathrm{pa}(X)$ represents the parents of a node variable $X$.

The factorization for the JPD is based on the independence properties obtained from the $\mathrm{BN}$ structure and the chain rule for the PGMs [9]. In this case study, $\mathrm{pa}(\mathrm{C} 1)=\varnothing$ (i.e. the empty set) and $\mathrm{pa}(\mathrm{C} 2)=\mathrm{C} 1$. In real-world systems there could be several components with functional dependencies that collectively implement a single system function. In such cases the $\mathrm{BN}$ might consist of several nodes and edges. Relevant examples of factorization for a $\mathrm{BN}$ with more than two nodes is given in [1], [8]. The JPD table for all possible state spaces for node variables $\mathrm{C} 1$ and $\mathrm{C} 2$ is provided in Table II.

\section{Bayesian Inference and Queries}

One of the main applications of BN in risk analysis is the ability to estimate the likelihood of various queries, as more evidence is available [8]. A possible query for the $\mathrm{BN}$ of the case study model could be "What is the probability of an intolerable (high) impact on the system due to EMI effects on C2, given that the EMI effects on C1 are tolerable (low)?". Using conditional probability, this query can be expressed as:

$$
P(C 2=\text { high } \mid C 1=\text { low })=\frac{P(C 1, C 2)}{P(C 1)}=0.023
$$

TABLE II. JoINT ProbabILITy Distribution TABLE FOR SEVERITY OF EMI IMPACT FOR C1 AND C2

\begin{tabular}{llll}
\hline \hline P(C1, C2 $)$ & C2 $=$ low & C2 $=$ medium & $\mathrm{C} 2=$ high \\
\hline $\mathrm{C} 1=$ low & 0.942839 & 0.012933 & 0.022513 \\
$\mathrm{C} 1=$ medium & 0.001277 & 0.010378 & 0.000479 \\
$\mathrm{C} 1=$ high & 0.00016 & 0.001916 & 0.007504 \\
\hline \hline
\end{tabular}

Further inference queries related to either a single node variable or to a specific group of node variables in the BN can be answered by the method of marginalization, which is also known as sum-product variable elimination [4]. For the case study, the query "What is the probability of a tolerable (low) impact on the system due to EMI effects on C2?" can be obtained by marginalizing $\mathrm{P}(\mathrm{C} 2=l o w)$, i.e., by performing a summation of all values corresponding to the JPD column $\mathrm{C} 2=$ low in Table II.

The JPD table values are actually the product of terms given in (6). The marginal probabilities of $\mathrm{C} 2=l o w$, $\mathrm{C} 2=$ medium, and $\mathrm{C} 2=$ high are obtained as follows:

$$
\begin{gathered}
\sum_{C 1} P(C 1) P(C 2=\text { low } \mid C 1)=0.944276 \\
\sum_{C 1} P(C 1) P(C 2=\text { medium } \mid C 1)=0.025227 \\
\sum_{C 1} P(C 1) P(C 2=\text { high } \mid C 1)=0.030496
\end{gathered}
$$

It can be seen that the independent probability values $\mathrm{P}(\mathrm{C} 2)$ given in Table I and the marginal probability values for $\mathrm{P}(\mathrm{C} 2)$ obtained from the Bayesian inference of (8)-(10) are different. This is because the latter consider the functional dependence of $\mathrm{C} 2$ on $\mathrm{C} 1$, facilitated by the BN. Since the case study has just two nodes in the BN model, the inferences are quick and do not necessarily need thorough mathematical formalization. In practice, however, for more realistic systems with multiple components and component failure states, a similar BN would have many nodes and edges, resulting in an exponential increase in the order of the JPD.

In such cases, some subexpressions in the joint distribution that need be estimated for specific probability queries depend on a small number of variables, according to the independencies provided by the BN structure [4], [7] . Other inference techniques such as maximum a posteriori (MAP) inference can be further used during the risk management process to find the single highest probability. In the current analysis, a MAP query could be "What is the probability value for the most likely impact severity class due to EMI effects on $C 2$ given that EMI effects on C1's are intolerable?". From the CPD table of Fig. 3 for node $\mathrm{C} 2$, it can be seen that the highest probability value given that $\mathrm{C} 1$ is high is 0.78 , which occurs when $\mathrm{C} 2$ is also high. The mathematical formulation of MAP queries for complex system is described in [4].

\section{MERITS AND LIMITATIONS OF PGMS IN RISK ANALYSIS}

Risk analysis for complex systems (including road vehicles, aircraft, ships etc.) involves multiple system functions, components and failure states. For such systems, risk analysis with PGMs like BN and Markov Random Fields [10] provide better handling of the system complexity, through graphical visualization of the system components/subsystems together with their functional dependence in a single model.

Some of the advantages of using PGMs over the traditional risk analysis tools such as event tree analysis (ETA) and fault tree analysis (FTA), for risk estimation include the following.

1) The structures of fault trees and event trees are unidirectional, with the result that analysing common cause failures with bidirectional functional dependencies between 
components would require multiple tree structures for a single system. Using PGMs, however, bidirectional functional dependencies can be represented in a single system model.

2) Compact representations of complex distributions, mathematical formalizations for inference tasks, and software tools for PGM realization, can support the decision-making process by making these process be more efficient and less time consuming.

3) Other technical and non-technical aspects relating to a component that may influence its EM susceptibility (such as component location within the system, shielding effects due to surrounding structures, EMI detection measures etc.) may also be added as additional nodes to the PGM structure. Further details on this are provided in [8].

The limitations of BN include modelling and computing difficulties as the number of parents associated with any single node in the model increases. For example, if a component $\mathrm{X}$ depends on ten other components or node variables, then the CPD table for node $X$ would have $2^{10}=1024$ entries. This would be a difficult task for an expert to perform, without access to sufficient data. In such cases, however, possibility theory or fuzzy logic could be employed to assign the CPD values [1]. Also, BNs belong to a class of PGMs having directed acyclic graph structures. Thus, BNs are unable to represent functional dependencies involving feedback loops. A possible solution to this limitation is to employ another class of PGMs called Markov random fields, which are graphs with undirected cyclic structure [10], thereby permitting feedback loop type dependencies. Another potential solution (although with increased complexity) is to employ dynamic BNs, which are temporal PGMs, modelled in time-slices [4].

\section{CONCLUSIONS AND FUTURE WORK}

For complex systems, estimating the risk of EMI impact on the system functions involves considerable epistemic uncertainty arising from the lack of detailed system knowledge. Moreover, EMI being a common cause of failure, it can simultaneously affect more than one component or subsystem, with the potential to cause even more severe system-level consequences due to the functional dependence between the affected components and/or subsystems.

In this paper, representative probabilistic data relating to EMI impact was generated using very simple methods in order to allow the CPD entries for a very simple BN model to be constructed for the case study outlined in Section II. In practice, however, such data could be derived from more realistic and detailed physical measurements or numerical simulations, or a mix of measurements and simulations.

This synthetic data allowed the identification of the severity of the system-level consequences, and hence estimation of the EMI-related risk, to be illustrated for a very simple system with some functional dependence. In addition, this data allowed the demonstration of various inference techniques that could be used to formulate and answer probability queries that would help to support system risk assessments.

Future work will further extend and develop the use of PGMs for system risk analysis, such as combining data obtained from measurements and/or simulations with the influence of non-technical factors on the key risk parameters (likelihood and severity). In addition, related automotive applications, such as communications system performance, will be investigated using this type of analysis.

\section{APPENDIX - GENERATION OF STATISTICAL DATA}

Firstly, it should be noted that the EMI probability distributions used in this work reflect neither the detailed physics of EMI nor the practical methods used to mitigate EMI effects, both of which are outside the scope of this paper. The results described here are purely synthetic and solely for the purposes of providing a representative source of statistical data for illustrating the use of PGM methods that are the subject of this paper.

Determining the likelihood of EMI in components $\mathrm{C} 1$ and C2 having a particular severity of system level impact involves determining the probability and magnitude of the deviation from the intended output due to EMI noise in signals $m_{1}$ and $m_{2}$. This Appendix details of details the determination of $G_{k}\left(E_{l}\right)$ and $G_{k}\left(E_{2}\right)$ that are used for calculating the relative deviations $D_{k}\left(E_{l}\right)$ and $D_{k}\left(E_{2}\right)$ of Section III.B with reference to a specific target value, $F=150$, and with a particular EMI noise example $N_{a}$ (with amplitude $1.38 \mathrm{~V}$, frequency 20.58 $\mathrm{kHz}$ and phase 5.5 radians) affecting $\mathrm{C} 1$ and/or $\mathrm{C} 2$.

\section{A. EMI Condition $E_{1}\left(m_{1}+N_{a}\right)$}

Addition of noise to signal $m_{l}$ (i.e. affecting $\mathrm{C} 1$ only) is considered as the first potential EMI condition, $E_{1}$ (see Section III.B). In practice it is not actually necessary to include the PWM modulation and demodulation as indicated in (2) since it is assumed that $\mathrm{C} 2$ is not affected by EMI in this case.

The digital signal $m_{l}$, obtained by converting the target value $F=150$ to a rectangular pulse-train in binary format corresponding to ' 10010110 ' as shown by the input in Fig. 5(a). The noise signal $N_{a}$ is then simply added to the

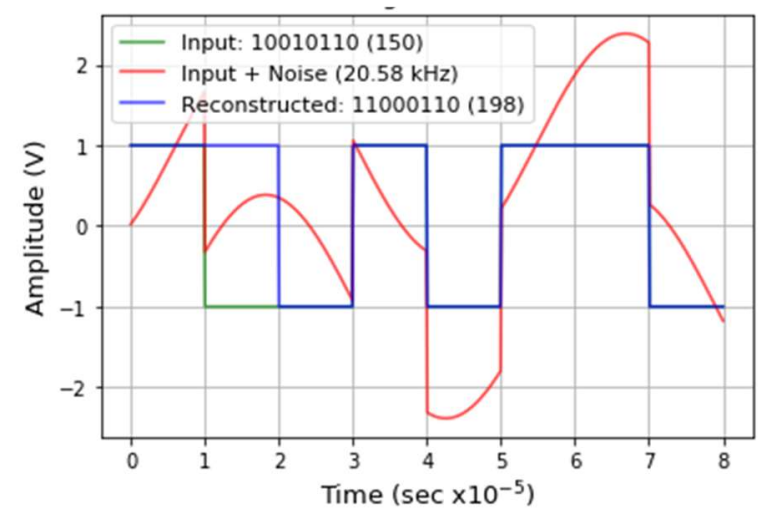

(a)

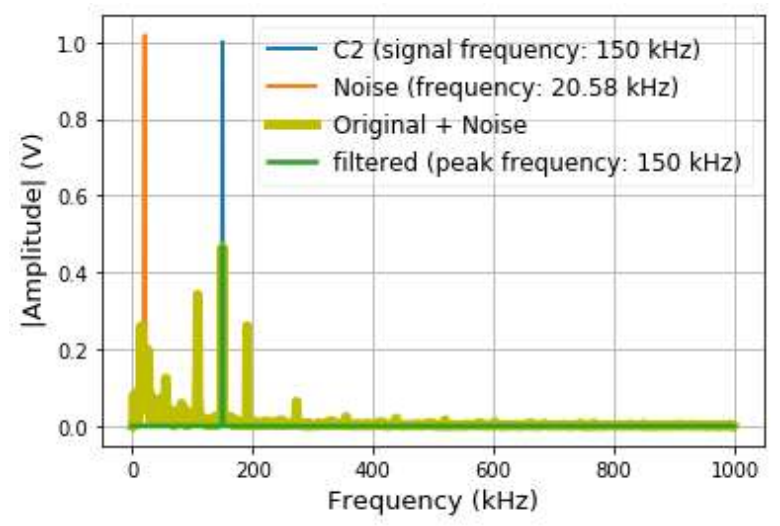

(b)

Fig. 5. Original and corrupted signals (due to noise sample $N_{a}$ ) for EMI conditions (a) $E_{1}$ and (b) $E_{2}$. 
signal $m_{1}$ to obtain the input + Noise waveform in Fig. 5(a). It can be observed that the addition of noise completely disrupts the intended signal. Nevertheless, for the purpose of calculating the deviation, the noisy signal was assumed to be reconstructed and decoded at $\mathrm{S} 1$. To reconstruct the noisy signal, the mean amplitude value over one-bit time interval $\left(10^{-5} \mathrm{~s}\right)$ is compared to $0 \mathrm{~V}$. If the mean $>0 \mathrm{~V}$ then the bit value is $+1 \mathrm{~V}$, otherwise it is considered to be $-1 \mathrm{~V}$. The reconstructed 8-bit signal for $\left(N_{a}+m_{1}\right)$ is '11000110' which corresponds to a decimal value of 198 . So, for this example, using (1), the relative deviation $D_{a}\left(E_{1}\right)$ from the intended target value (150) is $32 \%$.

\section{B. EMI Condition $E_{2}\left(m_{1}+N_{a}\right)$}

Similarly, to condition $E_{1}$, the addition of noise to PWM signal $m_{2}$ (modulated based on a sinusoid of target frequency $150)$ is considered as the second EMI condition $E_{2}$. The steps to calculate of the deviation from target frequency is given below:

1) Generate a sinusoid of frequency $F=150 \mathrm{kHz}$ and then convert it into a PWM signal $m_{2}=\operatorname{Pwm}(150)$, shown in Fig. 6(a).

2) Add noise $N_{a}$ to signal $m_{2}$, as illustrated in Fig. 6(b).

3) Demodulate the corrupted signal $\left(N_{a}+m_{2}\right)$ to obtain the demodulated signal $\operatorname{Ipwm}\left(N_{a}+m_{2}\right)$, which is shown in Fig. 6(c).

4) Estimate the sinusoidal signal from the demodulated noisy PWM signal $\left(N_{a}+m_{2}\right)$ by selecting the highest peak from the Fourier transform of the demodulated signal $\operatorname{Ipwm}\left(N_{a}+m_{2}\right)$.

5) Determine the difference between the frequency value obtained from this process and the original target frequency of $150 \mathrm{kHz}$.

For this example, the noise waveform $\left(N_{a}\right)$ yields a relative deviation $D_{a}\left(E_{2}\right)=0$.

C. EMI condition $E_{3}\left(E_{1}+E_{2}\right)$

In EMI condition $E_{3}$, condition $E_{1}$ is first applied to signal $m_{1}$ and then the decoded value is subjected to PWM modulation before the application of EMI condition $E_{2}$. The resulting difference $D_{a}\left(E_{3}\right)$ is then obtained from the peak of the Fourier transform of the demodulated signal received at $\mathrm{S} 2$, as indicated in (4).

\section{REFERENCES}

[1] L. Devaraj, A. R. Ruddle and A. P. Duffy, "System level risk analysis for immunity in automotive functional safety analyses," Proc. 2020 International Symposium on Electromagnetic Compatibility - EMC Europe 2020, Rome, Italy, 2020.

[2] L. Devaraj, A. Ruddle, A. Duffy, "Electromagnetic risk analysis for EMI impact on functional safety with probabilistic graphical models and fuzzy logic," IEEE Letters on Electromagnetic Compatibility Practice and Applications, vol. 2, No. 4, December 2020, pp. 96-100.

[3] E. Genender, H. Garbe and F. Sabath, "Probabilistic risk analysis technique of intentional electromagnetic interference at system level," IEEE Trans. Electromag. Compat., vol. 56, (1), pp. 200-207, 2014.

[4] D. Koller and N. Friedman, Probabilistic Graphical Models: Principles and Techniques, MIT Press, London; Cambridge, Massachusetts, 2009.

[5] P. Weber et al, "Overview on Bayesian networks applications for dependability, risk analysis and maintenance areas," Engineering Applications of Artificial Intelligence, vol. 25, (4), pp. 671-682, 2012.

[6] S. Yang et al, "Study on the EMI impact over the safety of railway signaling and case analysis," 2017 2nd International Conference on System Reliability and Safety, vol. 2018, pp. 374-379, Milan, Italy, Dec. 2017

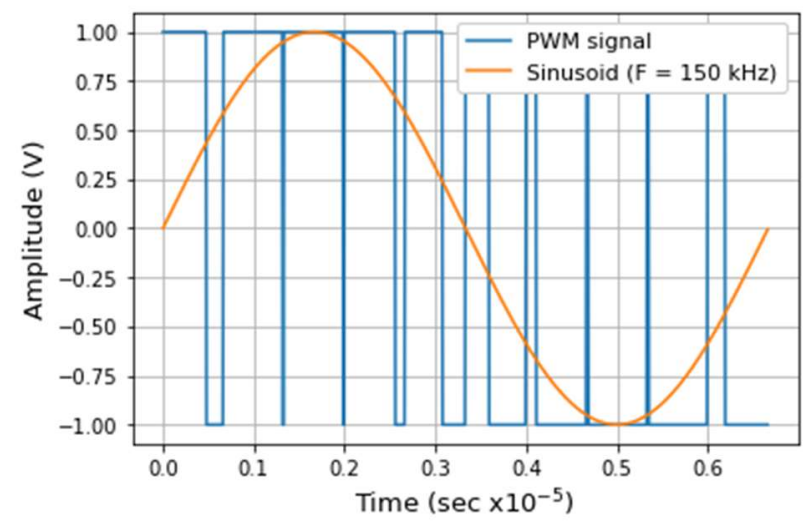

(a)

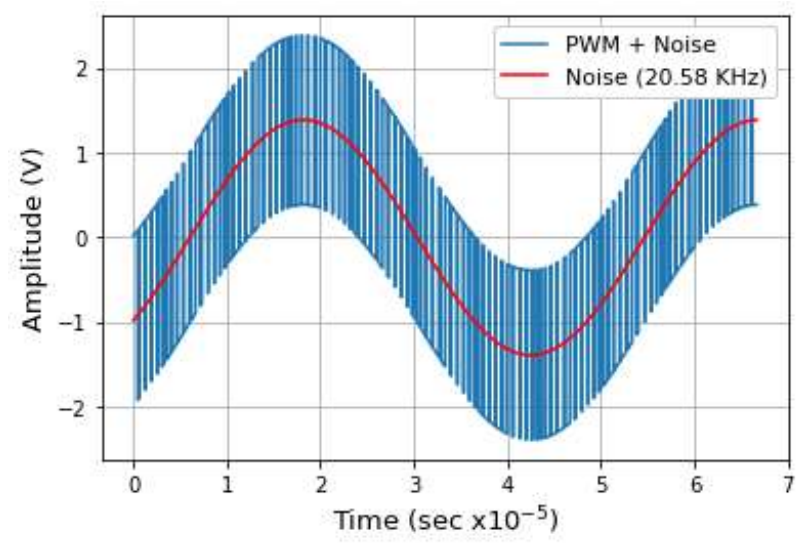

(b)

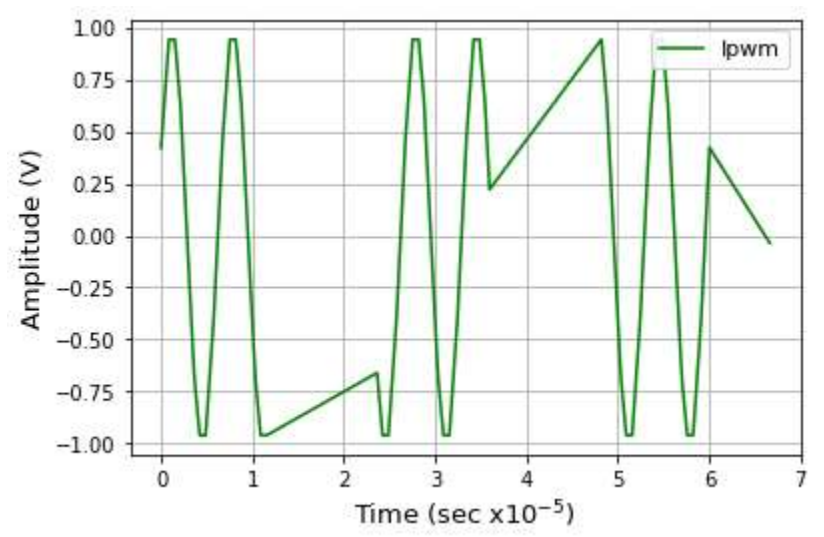

(c)

Fig. 6. Example plots to illustrate steps considered in EMI condition $E_{2}$ : (a) target frequency $F$ and $\operatorname{Pwm}(F)$ waveform; (b) noise $N_{a}$ and waveform $\operatorname{Pwm}(F)+N_{a}$; and (c) demodulated noisy waveform $\operatorname{Ipwm}\left(\operatorname{Pwm}(F)+N_{a}\right)$

[7] N.G. Leveson, Engineering a Safer World - Systems Thinking Applied to Safety, MIT Press, Cambridge, MA, 2011.

[8] L. Devaraj, A. R. Ruddle, A. P. Duffy and A. J. M. Martin, "Riskassessment approach for EM resilience in complex systems using Bayesian Networks," Proc. 2021 Joint IEEE Int. Symp. Electromag. Compat., Signal \& Power Integrity, EMC Europe 2021, Sept. 2021.

[9] A. Freno and E. Trentin, Hybrid Random Fields: A Scalable Approach to Structure and Parameter Learning in Probabilistic Graphical Models, Intelligent Systems Reference Library Vol. 15, Springer-Verlag, Berlin, ISBN 978-3-642-20307-7, pp. 28-33, 2011.

[10] Wainwright M J, Jordan M I (2008), "Graphical models, exponential families, and variational inference," in Foundations and Trends in Machine Learning, vol. 1, Nos. 1-2, pp. 1-13. 